\title{
Natural selection and population dynamics
}

\author{
llik Saccheri ${ }^{1}$ and Ilkka Hanski ${ }^{2}$
}

\begin{abstract}
${ }^{1}$ School of Biological Sciences, University of Liverpool, The Biosciences Building, Crown Street, Liverpool, UK, L69 7ZB
${ }^{2}$ Department of Biological and Environmental Sciences, PO Box 65 (Viikinkaari 1), FI-00014 University of Helsinki, Finland
\end{abstract}

\begin{abstract}
To what extent, and under which circumstances, are population dynamics influenced by concurrent natural selection? Density dependence and environmental stochasticity are generally expected to subsume any selective modulation of population growth rate, but theoretical considerations point to conditions under which selection can have an appreciable impact on population dynamics. By contrast, empirical research has barely scratched the surface of this fundamental question in population biology. Here, we present a diverse body of mostly empirical evidence that demonstrates how selection can influence population dynamics, including studies of small populations, metapopulations, cyclical populations and host-pathogen interactions. We also discuss the utility, in this context, of inferences from molecular genetic data, placing them within the broader framework of quantitative genetics and life-history evolution.
\end{abstract}

\section{Introduction}

That genetically based individual variation in life-history traits could influence population dynamics has interested population biologists since at least 1952, when Dennis Chitty proposed a mechanism involving natural selection to explain population cycles in field voles Microtus agrestis ([1], reviewed in [2]). Previously, in 1930, Fisher [3] concluded that, under the idealised conditions of a constant environment, natural selection increases the growth rate of a population, but that, in practice, environmental deterioration owing to physical and biotic changes, including increased population density, has the opposite effect, such that the intrinsic rate of increase fluctuates around zero. Thus, although natural selection often determines which individuals survive and reproduce, the actual number of individuals that survive is generally determined by one or more external limiting factors, such as food, space or predation [4]. For instance, even strong selection on any trait is unlikely to make a marked difference to population dynamics in a holenesting bird population that is limited by the availability of nest sites. However, there are other situations where a link between selection and population dynamics is more likely. For instance, in a butterfly metapopulation (see Glossary) persisting in a balance between local extinctions

Corresponding author: Saccheri, I. (saccheri@liv.ac.uk).

Available online 18 April 2006 and colonisations in a fragmented habitat, selection on traits influencing the migration of independently dispersing individuals will have (meta)population dynamic consequences via its impact on the rate of fragment recolonisation.

\section{Theoretical framework}

Charlesworth presented a general quantitative framework relevant to studying the consequences of selection on population size, taking into account age-specific and density-dependent survival and fecundity [5]. In this framework, it becomes clear that the population dynamic response can be critically sensitive to selection on some specific life-history components, but not on others. This approach draws broadly from population ecology and evolutionary biology; historically, however, the interplay between selection and population dynamics has been approached from three complementary and overlapping perspectives. The first is that of population ecologists, who have recognised the need to incorporate individual variation in models of population regulation $[1,2,6,7]$. The second is that of population geneticists, who extended

\footnotetext{
Glossary

Additive genetic variance: the component of the phenotypic variance for a trait that is due to the additive effects of genes; it is the chief cause of resemblance between relatives and determines the response of the population to selection. Association mapping: a general method of locating the chromosomal region of genes affecting a trait of interest by looking for associations between a large panel of genetic markers and phenotypic variation in the trait.

Candidate gene approach: a strategy to identify genes affecting a trait of interest by focusing on genes known to have a related function in other organisms.

Genetic drift: random fluctuations in allele frequency owing to finite sampling of gametes, generally resulting in reduced genetic variation and the eventual fixation of one allele within any given population.

Inbreeding depression: reduction in mean fitness or any of its components in the offspring arising from reproduction between relatives.

Linkage disequilibrium: the nonrandom association of alleles at two or more loci. It can arise by chance through genetic drift, or through selection for a particular multi-locus combination.

Metapopulation: a network of often small but reproductively more or less independent local populations (often called demes) connected by migration. Mutational meltdown: a hypothetical process whereby the accumulation of mildly deleterious mutations through genetic drift leads to a declining population growth rate, causing further drift and mutation accumulation, ultimately sending the population to extinction.

Overdominance (or heterozygote advantage): the case in which the heterozygote genotype has a higher fitness than either the homozygote dominant or the homozygote recessive genotype.

Pleiotropy: when a gene affects more than one phenotypic trait.

Virulence: the capacity of a pathogen to cause disease, expressed as percentage mortality of infected hosts.
} 
the basic population genetics models to density-regulated populations, ostensibly to explore consequences for genotype frequencies but, in so doing, also revealing possible reciprocal effects of such evolutionary changes on population size [8]. The third approach is that of evolutionary biologists wishing to understand life-history dynamics in relation to environmental conditions, including population density [9], through the assessment of tradeoffs within the limits of the available additive genetic (co)variance [10]. There is a clear trend toward the increasing integration of these perspectives [11-13], reflected by a growing acceptance that the traditional dichotomy between ecological and evolutionary timescales is a false one.

The issue of timescale is nonetheless relevant while considering the role of natural selection in population dynamics. It is evident that life histories affect population dynamics; life histories evolve and, in this sense, selection affects population dynamics. But we are more concerned here with population dynamics being affected by concurrent selection. To demonstrate that such selection affects population dynamics requires, minimally, the simultaneous measurement of: (i) density in spatially or temporally replicated samples; (ii) any ecological factors affecting population regulation and density; and (iii) selection on genetic variation for demographically significant traits. The simultaneous measurement of (i)-(iii) is a formidable logistic challenge in all but the simplest systems, particularly as many components of a life-history potentially influence population growth rate. Elasticity analysis [14] is an effective tool for evaluating the relative importance of different phenotypic traits on population growth rate through their effects on survival and reproduction. Some generalisations that are relevant to particular taxa should emerge from such analyses, bearing in mind that dissimilar ecological circumstances can alter the influence of particular traits even within a single species [15].

\section{Conservation biology and the extinction vortex}

The potential consequences of genetic factors on population growth rate and extinction risk were brought to prominence during the 1980s through the influential conservation biology texts by Otto Frankel and Michael Soule [16-18]. At that time, the focus of conservation biology was on the persistence of small populations and, therefore, the effects of inbreeding depression and reduced adaptive potential through genetic drift ('genetic erosion') were widely discussed. These considerations were stimulated by the idea of an 'extinction vortex' generated by a positive feedback between declining population growth rate and genetic erosion [17]. Lande [19] reminded us that these genetic factors cannot be treated in isolation of the ecological context (i.e. population regulation), and he concluded that genetic factors are generally of secondary importance in setting minimum viable population sizes. This conclusion appeared to challenge the significance of genetic factors in biological conservation (reviewed in [20]).

\section{Inbreeding depression in natural populations}

Until recently, inbreeding depression has received greater emphasis than has adaptive genetic variation as a candidate for genetic impacts on demography. This makes sense, to the extent that fitness is generally expected to show an immediate decline with inbreeding through the effects of deleterious homozygotes, whereas the fitness consequences of life-history variants are likely to be more dependent on the ecological context and, therefore, more difficult to detect. A few longitudinal field studies of island populations involving near-complete pedigrees have proved invaluable for studying interactions between inbreeding and environmental stress. The first of these, by Keller et al. in 1994 [21], found that less-inbred song sparrows Melospiza melodia had higher probability of surviving a severe storm. In the same year, Jimenez et al. demonstrated that the cost of inbreeding (reduced survival) in mice is likely to be greater in natural than in benign captive environments [22]. Keller and Waller's compilation of cases of inbreeding depression in natural populations includes a diverse set of animals and plants [23]. Components of fitness that are likely to exhibit strong inbreeding depression include fertility, fecundity, juvenile and larval viability and competitive ability [24]. Generally, the severity of inbreeding effects is expected to increase sharply under conditions of environmental stress, as illustrated by parasite infection in Soay sheep Ovis aries [25] and high temperatures in Drosophila melanogaster [26].

The first demonstration of inbreeding increasing the risk of population extinction in a natural system came from a large-scale study more focused on the ecology than on the genetics of the large metapopulation of the Glanville fritillary butterfly Melitaea cinxia [27]. Further evidence comes from a simple but effective field experiment in the outcrossing plant Clarkia pulchella [28]. What is striking about this study is that a modest $10 \%$ inbreeding differential among populations led to a $60 \%$ increase in extinction risk. In other studies, the demographic effects of inbreeding have been revealed through recoveries of previously inbred populations owing to natural or experimental genetic rescue (reviewed in [20]).

\section{Population regulation: hard versus soft selection}

The question about population regulation occupied the minds of population ecologists for decades and led to occasionally heated debates [29]. For most ecologists, the matter is now straightforward: some density dependence in population dynamics is necessary to enable the longterm persistence of populations, although the strength and temporal and spatial scales in the occurrence of density dependence can vary greatly [30,31]. Generally, most, although not all, vertebrate populations are relatively tightly regulated, whereas many insect populations fluctuate wildly and give the impression of weak regulation [31]. During the 1990s, ecologists realised that many species inhabiting fragmented habitats occur in metapopulations with weakly regulated and ephemeral local populations, in which case much of the regulation occurs at the level of the entire metapopulation rather than at the level of local populations [32]. 
The type of population regulation relates to the concepts of hard and soft selection in population genetics (Box 1). In the metapopulation context, soft selection implies local population regulation, whereas hard selection is associated with global regulation: each local population contributes to the next generation in proportion to the mean fitness of individuals, as determined by their genotypes [33]. Entirely global regulation is not possible in real metapopulations [34], but regulation can be largely global, leading to hard selection. We could therefore expect to find examples of selection influencing population dynamics especially in species that persist as metapopulations, and indeed such examples have been documented (Box 2).

\section{Soft selection and population cycles}

Do we have evidence for soft selection affecting population dynamics? Although it has been shown that, in principle, density-dependent selection can generate regular oscillations in population size and allele frequency [8], there was until recently no direct empirical support from any natural system. The classic papers by Chitty ([1] and others) envisioned natural selection explaining regular population cycles of boreal and arctic voles, but the

\section{Box 1. Hard and soft selection}

The tradition in population genetics is to assume that, for any given environment in the absence of information to the contrary, fitnesses are independent of population density and of the frequencies of other genotypes. In reality, this is unlikely to be so in strongly regulated local populations. To take the consequences of local competition into account and, in so doing, explain how populations can sustain high genetic loads (i.e. the burden of suboptimal genotypes) without going extinct, Bruce Wallace introduced the concept of hard and soft selection $[66,67]$. Soft selection refers to situations where the strengths of selection coefficients $s$ are density and frequency dependent; hard selection refers to cases where the values of $s$ are independent of both. Additionally, $s$ can also be density or frequency dependent.

The relevance of these concepts to the influence of natural selection on population regulation is that hard selection causes mortality independently of the factors related to local density (or genotype frequencies), be they selective or not, and might therefore have a direct effect on population size. The other density-dependent agents of population regulation might also compensate for the effects of such hard selection, depending on when during the life cycle it occurs, and on its magnitude [8]. For example, the same level of hard selection imposed by an extreme temperature shock is more likely to impact population growth rate when applied to adults (representing the survivors of density-dependent factors) than to juveniles. Densitydependent or soft selection, however, removes individuals that would die or fail to reproduce anyway, for reasons unrelated to their genotype, ideally with no effect on population size (Figure la). However, conditions do exist under which soft selection can affect population size, for instance through competition between genotypes whose reproductive rates are differentially sensitive to their own density $[8,36]$ or to that of a predator [37]. Soft and hard selection can also be viewed in terms of local (within-population) and global (between-population) selection in the context of a metapopulation, with corresponding effects on the effective size of local populations (Figure lb). Empirical data on the relative strengths of soft versus hard selection are limited [68]. One should also recognise that hard and soft selection represent extremes of a continuum, and, in practice, that natural selection is a mix of the two. (a)

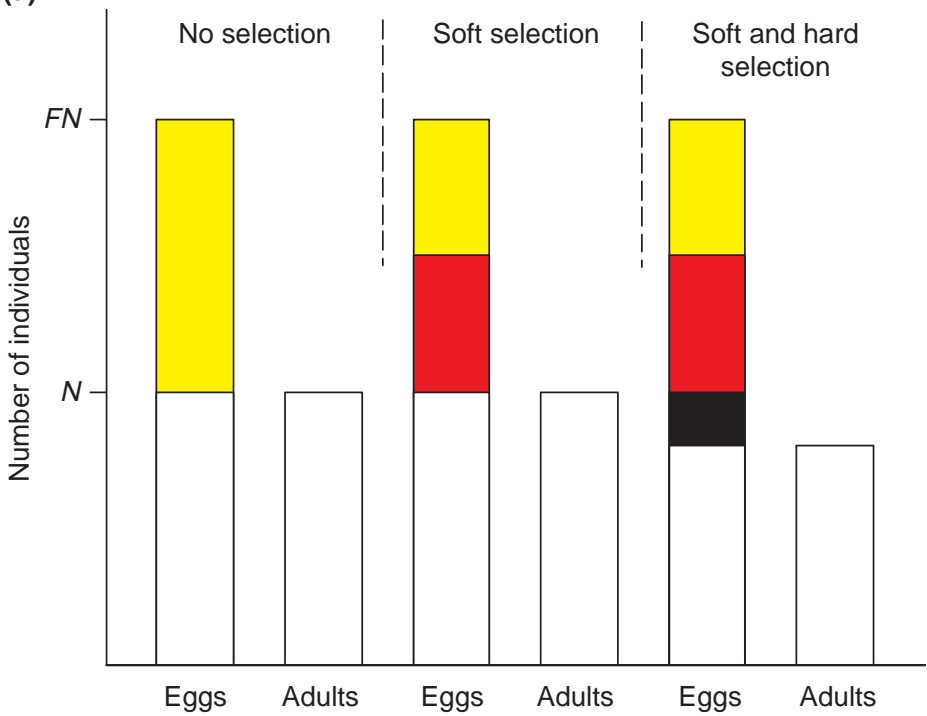

(b)

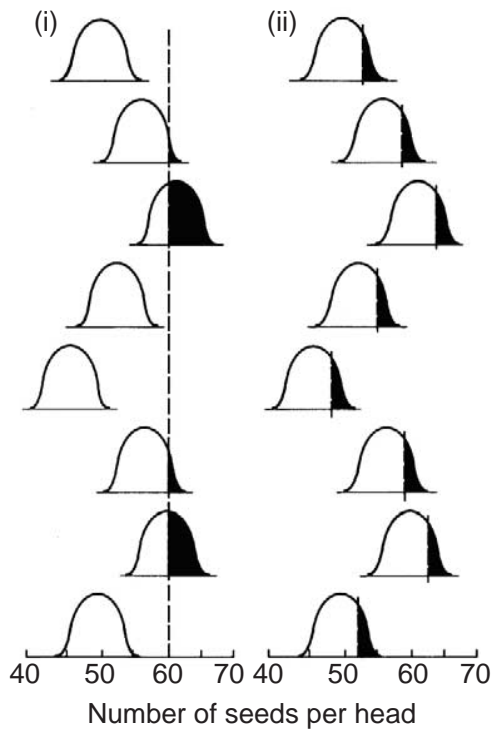

Figure I. Representations of hard and soft selection. (a) The concept of hard and soft selection (with respect to population density only, for simplicity not showing frequency dependence). A population of $N$ adults with average fecundity of $2 F$ per female produces $F N$ eggs. As maximum adult population size is limited to $N$ by some external factor, such as availability of space or food, $2 F-N$ eggs must die each generation. This mortality can be random with respect to genotype and, therefore, nonselective (in yellow), or selective (soft selection, in red), but cannot depress population size below $N$. Hard selection (in black) introduces an additional, densityindependent source of mortality, which reduces population size below $N$. (b) An early representation of hard versus soft selection in the context of a metapopulation. In (i), the small-grain breeder saves all heads bearing 60 or more seeds for planting and future selection; some experimental plots are entirely discarded under this scheme. In (ii), the breeder first samples a few heads from plants of each experimental plot, determines the statistical distribution of seeds per head for each plot, and then harvests what is estimated to be the best $5 \%$ of all heads of each plot for planting and further selection. Under this scheme, a few heads are saved from each plot regardless of its average number of seeds per head. Soft selection resembles the second scheme, hard selection the first. Black shading indicates those individuals that were selected to reproduce, no shading those that were not selected. Modified, with permission, from [69] (a) and [67] (b). 


\section{Box 2. Hard selection in a butterfly metapopulation}

Studies of the large metapopulation of the Glanville fritillary butterfly Melitaea cinxia in Åland, southwest Finland, have detected hard (between-deme) selection (Box 1) in relation to several components of fitness. In this butterfly metapopulation, which is characterised by high rates of local extinction compensated for by the recolonisation of currently empty habitat patches, extinction risk is strongly influenced by population size per se, immigration from neighbouring populations (the rescue effect), climatically driven spatially correlated density fluctuations, and other ecological and environmental processes $[32,70]$. In addition, elevated inbreeding in small populations further increases the extinction risk [27]. An important message from this study is that, even in situations where environmental and demographic stochasticities dominate, selection against inbred genotypes can increase extinction risk.

Migration leading to gene flow in metapopulations is the process that enables populations consisting of superior genotypes to increase their share in the metapopulation as a whole. This leads to a mixture of local- and metapopulation-level selection, a syndrome dubbed the 'metapopulation effect' [71]. In the Glanville fritillary, migration leads to the assortment of individuals with genetically determined more and less dispersive phenotypes among new versus old populations [72]. Recent work on this species has shown that there are significant differences among individuals in terms of their flight metabolic rate, flight performance and fecundity associated with a single gene polymorphism (the glycolytic enzyme phosphoglucose isomerase Pgi [73]). Furthermore, population growth rate is also affected by the allelic composition of Pgi, although in a manner that is specific to the degree of isolation and area of habitat patches [65], owing to complex interactions between landscape structure and the performance of individuals with different phenotypes. This is a clear example of molecular-level variation influencing population dynamics. Another example in the same butterfly metapopulation arises from heritable variation in female host-plant oviposition preference. In brief, the correspondence between the host-plant preference of migrating females and the host-plant composition of potential target meadows has a strong and significant effect on colonisation rate [74], while the extinctioncolonisation dynamics appear, in turn, to influence the evolution of host-plant preference in the metapopulation [75].

consensus now is that selection is not involved in vole dynamics [35]. Fifty years on, two independent studies, on species other than voles, [36,37], revealed a key role for genetic variation and soft selection in population density cycles. Sinervo et al. [36] showed that two-year adult density cycles in natural populations of the side-blotched lizard Uta stansburiana are due to alternating densityand frequency-dependent selection for two contrasting life-history strategies. At low density, $r$-type orangethroated females lay many small eggs, outcompeting $K$-type yellow-throated females, which lay relatively fewer but larger eggs, leading to high adult density and high orange morph frequency in the following year. At high density, although relatively rare, the larger, more competitive offspring produced by yellow-throated females gain the upper hand; adult population density subsequently declines and yellow morph frequency increases. Just as delayed density dependence is the key to population cycles in the absence of evolutionary dynamics, the time lag between soft (density- and frequencydependent) selection on juveniles and the change in the intrinsic growth rate is what produces population oscillations in this system.

What would happen to the population cycles in the absence of life-history variation? In an elegant experiment, Yoshida et al. [37] addressed this question by manipulating the diversity of algal clones (life-history variation) in a predator-prey (rotifer-alga) system. Their results show that, in the absence of genetic variation, the two species oscillate as predicted by classic predator-prey models [38], whereas genetic diversity in the prey population fundamentally alters the dynamics, resulting in much longer cycles for both species that are completely out of phase with each other. Again, the explanation involves a delayed density response, in this case of the grazing predator with respect to abundance and nutritional quality of the algal population, which undergoes rapid cyclical evolution in relation to grazing pressure. This example illustrates how, through interspecific competition, selection in one species can affect population dynamics in another (Box 3).

\section{Genetic mechanisms and constraints}

The response to selection on any specific trait can only be fully understood in the context of the entire life history and the underlying genetic architecture. Selection coefficients can differ between age classes and the response of one trait could be influenced by selection on another with which it is genetically correlated, owing to pleiotropy or linkage disequilibrium. Therefore, hypotheses linking population dynamics to selection in natural populations need to consider explicitly the mechanistic basis of the selection response. Quantitative genetic theory of lifehistory evolution [10] provides a statistical framework for studying the evolutionary dynamics of complex phenotypes determined by many genes. The essential genetic parameters required for this approach are those describing the genetic variance-covariance matrix, estimated from phenotypic measurements on relatives and artificially selected lines [39].

As we discuss here, research projects are increasingly using molecular genetic techniques to characterise genetic

\section{Box 3. Evolution affecting abundance in host-pathogen systems}

The general question about how evolutionary dynamics within a community of two or more interacting species might influence abundances has been studied theoretically for a variety of standard ecological interactions, including interspecific competition, predator-prey, host-pathogen, and plant-herbivore-carnivore interactions $[76,77]$.

The host-pathogen interaction is of particular interest because there is strong evidence that pathogens (bacteria, viruses, fungi, protozoa and parasites) can have a major regulatory influence on their host populations through high mortality [78]. Biological control of the European rabbit Oryctolagus cuniculus in Australia and Europe, through infection with myxoma virus, is a clear example of how the interplay between the evolutionary and population dynamics of pathogen and host can affect the abundance of both species [79]. The highly virulent strain of myxoma virus first introduced from South America during the early 1950s decimated rabbit populations in many parts of Australia and Europe, but was rapidly outcompeted by mutants of intermediate virulence, which gave more time for transmission by mosquito or flea vectors. On the host side, rabbit populations responded to the intense selection by evolving a degree of resistance to the pathogen. The result was partial recovery of rabbit populations, although the disease still has a considerable influence on their numbers [80]. 
and developmental mechanisms, often at the expense of quantitative genetic approaches. The latter approach is generally more efficient for studying polygenic variation and genetic correlations; the former for variation governed by few genes of large effect. The molecular approach also provides an alternative for characterizing genetic parameters in species that are difficult to breed in captivity. Where possible, the combined use of both approaches is likely to yield the most useful description of genetic architecture.

\section{The contribution of molecular genetics}

The rise of molecular genetics over the past 20 years has influenced substantially the way in which population ecology is conducted but, so far, has had only limited impact on our understanding of population regulation. The emphasis in molecular ecology has been on the application of neutral markers to describe population structure at different scales, information that can be used to make inferences about some demographic processes (e.g. gene flow [40] or population expansion and contraction [41]), but which on its own cannot contribute to a mechanistic understanding of population dynamics. Only recently have studies of genetic population structure begun to place greater emphasis on the need to incorporate actual data on density and other demographic parameters into analyses seeking to describe how particular patterns of genetic variation arise.

Over the same period, conservation genetics has produced information about inbreeding depression [23], theoretical elaborations on changes in additive genetic variance with inbreeding [42], theoretical models of minimum viable population size [43,44], the concept of 'mutational meltdown' [45], some applications of adaptive dynamics [13], and more data on population structure - all necessary and useful, but rarely addressing directly the question about selection and population dynamics. The disciplines of molecular genetics and conservation genetics have now matured, conceptually and technically, to the point where molecular genetic tools can make an important contribution to the selection-population dynamics question.

A relevant application of neutral markers is as a genealogical trace in the construction and validation of pedigrees or sibships [46]. Pedigrees coupled with lifehistory data are the ultimate resource for revealing variation in components of fitness, but are exceedingly difficult and labour intensive to follow in natural populations. Marker-assisted reconstruction will hopefully expand the sample of wild pedigrees, currently restricted to island populations of birds and ungulates, although samples involving overlapping generations remain problematic. Even in the absence of pedigrees, marker-inferred relatedness can be used to quantify genetic variation underlying traits of interest [47].

\section{Spatiotemporal analysis of adaptive genetic variation}

Several studies have demonstrated ongoing natural selection through changes in phenotype (genotype) frequencies over time and/or space, but few have investigated reciprocal effects of density and selection.
Classic examples include clines (e.g. Drosophila alcohol dehydrogenase [48]; melanism in the peppered moth Biston betularia [49]; and insecticide resistance in the mosquito Culex pipiens [50]) and hybrid zones (e.g. of the grasshopper Podisma pedestris, fire-bellied toads Bombina spp., and races of the butterfly Heliconius erato [51]). Selection is often expected to have no or minimal effect on density owing to density dependence, but explicit demonstrations of this assumption in relation to natural environments are few $[25,52]$.

Perhaps the greatest potential use of molecular genetics in this context is as a tool to score phenotypes that are usually difficult to detect directly in natural populations. This is only possible once genotype-phenotype correlations have been established, which is a nontrivial task. In addition to providing means to measure cryptic phenotypes, the advantage of PCR-based molecular assays (e.g. microsatellites or SNPs [53]) is that they can be applied efficiently to very large samples, often in a manner that is noninvasive to the population as a whole, thereby increasing the statistical power to detect individual-level genetic effects on demography across space and time. This general approach has so far been largely restricted to heterozygosity-performance correlations $[25,54,55]$. The implication of several studies [23] is that inbreeding depression is an important component of fitness variation in many natural populations, but it remains unclear to what extent such correlations reflect genome-wide expression of deleterious recessives, rampant overdominance or publication bias [56,57].

The search for ecologically important molecular genetic variation is not new [58] but it is fair to say that it is only now beginning to take off (e.g. $[59,60])$. Key demographic life-history traits for this effort will vary among species, but are likely to include components of mating success, fertility, fecundity, competitive ability, defence, disease resistance, stress tolerance and migration. A variety of strategies are being developed to apply association mapping to non-model organisms [61], and the candidate gene approach provides an increasingly viable alternative [62]. Large-scale assays of variable gene expression [63] provide another tool for characterizing the strength and scale of local adaptation, which could have population dynamic consequences. Combining cDNA microarrays with suppression subtractive hybridisation (SSH) [64] further extends our ability to identify differentially expressed genes underlying demographically relevant traits.

\section{Conclusions}

Many ecologists primarily interested in population dynamics have been sceptical of the relevance of genetics and natural selection in their research. Historically, this view stems from the belief that selection is invariably density dependent or soft, with negligible reciprocal effects on density itself. However, soft selection can generate population cycles [36,37], and conditions for hard selection might be relatively common, especially in fragmented landscapes $[27,28,65]$. Our own experience with the well-studied Glanville fritillary metapopulation, combined with several other lines of evidence, suggests 
that, until one looks carefully, it is unwise to assume that genetic variation and selection are irrelevant to population dynamics. The detection of such effects in other systems is likely to require large-scale longitudinal field studies with a focus on variation in demographically important life-history traits. The effect of environmental context and population density on selection coefficients will only be revealed through a greater appreciation of the spatial and temporal scale of environmental heterogeneity as experienced by individuals with well-characterised life histories. For populations and communities of species pushed beyond their past zones of relative comfort through anthropogenic environmental change, hard selection will increasingly become the hard reality.

Beyond its use for inferring population structure and demographic history, the value of molecular genetics in population ecology will be judged by the extent to which it provides fresh insight into the components of functional variation among individuals and facilitates the measurement of such variation in natural populations. Gene hunting might be an expensive luxury for ecologists but, incorporated into a framework of well-understood population ecology and life-history quantitative genetics, the potential dividends are great. The inverse of this argument is that, in our drive to characterise genetic and developmental architectures, we should not lose sight of the fact that fitness depends on the usually complex ecological context.

\section{Acknowledgements}

We thank M. Begon, R. Ennos, P. Hedrick, L. Keller, R. Lande and two anonymous referees for their helpful comments.

\section{References}

1 Chitty, D. (1952) Mortality among voles (Microtus agrestis) at Lake Vyrnwy, Montgomeryshire, in 1936-1939. Phil. Trans. R. Soc. B 263, $505-552$

2 Krebs, C.J. (1978) A review of the Chitty Hypothesis of population regulation. Can. J. Zool. 56, 2463-2480

3 Fisher, R.A. (1999) The Genetical Theory of Natural Selection: A Complete Variorum Edition, Oxford University Press

4 Haldane, J.B.S. (1953) Animal populations and their regulation. New Biol. 15, 9-24

5 Charlesworth, B. (1994) Evolution in Age-Structured Populations, Cambridge University Press

6 Birch, L.C. (1960) The genetic factor in population ecology. Am. Nat. 94, 5-24

7 Ginzburg, L.R. (1983) Theory of Natural Selection and Population Growth, Benjamin Cummins

8 Charlesworth, B. (1971) Selection in density-regulated populations. Ecology 52, 469-474

9 Cole, L.C. (1954) The population consequences of life history phenomena. Q. Rev. Biol. 29, 103-137

10 Lande, R. (1982) A quantitative genetic theory of life history evolution. Ecology 63, 607-615

11 Berry, R.J. et al., eds (1992) Genes in Ecology, Blackwell Scientific Publications

12 Silvertown, J. and Antonovics, J., eds (2001) Integrating Ecology and Evolution in a Spatial Context, Cambridge University Press

13 Ferrière, R. et al., eds (2004) Evolutionary Conservation Biology, Cambridge University Press

14 van Tienderen, P.H. (2000) Elasticities and the link between demographic and evolutionary dynamics. Ecology 81, 666-679

15 Coulson, T. et al. (2005) Decomposing the variation in population growth into contributions from multiple demographic rates. J. Anim. Ecol. 74, 789-801
16 Frankel, O.H. and Soulé, M.E. (1981) Conservation and Evolution, Cambridge University Press

17 Soulé, M.E., ed. (1986) Conservation Biology: The Science of Scarcity and Diversity, Sinauer Associates

18 Soulé, M.E., ed. (1987) Viable Populations for Conservation, Cambridge University Press

19 Lande, R. (1988) Genetics and demography in biological conservation. Science 241, 1455-1460

20 Frankham, R. (2005) Genetics and extinction. Biol. Conserv. 126, 131-140

21 Keller, L.F. et al. (1994) Selection against inbred song sparrows during a natural population bottleneck. Nature 372, 356-357

22 Jiménez, A.J. et al. (1994) An experimental study of inbreeding depression in a natural habitat. Science 266, 271-273

23 Keller, L.F. and Waller, D.M. (2002) Inbreeding effects in wild populations. Trends Ecol. Evol. 17, 230-241

24 Crnokrak, P. and Roff, D.A. (1995) Dominance variance: associations with selection and fitness. Heredity 75, 530-540

25 Coltman, D.W. et al. (1999) Parasite-mediated selection against inbred soay sheep in a free-living, island population. Evolution 53, 1259-1267

26 Bijlsma, R. et al. (1999) Environmental dependence of inbreeding depression and purging in Drosophila melanogaster. J. Evol. Biol. 12, 1125-1137

27 Saccheri, I. et al. (1998) Inbreeding and extinction in a butterfly metapopulation. Nature 392, 491-494

28 Newman, D. and Pilson, D. (1997) Increased probability of extinction due to decreased effective population size: experimental populations of Clarkia pulchella. Evolution 51, 354-362

29 Turchin, P. (1995) Population regulation: old arguments and a new synthesis. In Population Dynamics: New Approaches and Synthesis (Cappuccino, N. and Price, P.W., eds), pp. 19-40, Academic Press

30 Sinclair, A.R.E. (1989) Population regulation in animals. In Ecological Concepts (Cherrett, J.M., ed.), pp. 197-242, Blackwell

31 Hanski, I. (1990) Density dependence, regulation and variability in animal populations. Phil. Trans. R. Soc. B 330, 141-150

32 Hanski, I. (1999) Metapopulation Ecology, Oxford University Press

33 Whitlock, M.C. (2004) Selection and drift in metapopulations. In Ecology, Genetics, and Evolution in Metapopulations (Hanski, I. and Gaggiotti, O.E., eds), pp. 153-174, Elsevier

34 Hanski, I. et al. (1996) Random walks in a metapopulation: how much density dependence is necessary for long-term persistence? J. Anim. Ecol. 65, 274-282

35 Boonstra, R. and Boag, P.T. (1987) A test of the Chitty hypothesis: inheritance of life-history traits in meadow voles (Microtus pennsylvanicus). Evolution 41, 929-947

36 Sinervo, B. et al. (2000) Density cycles and an offspring quantity and quality game driven by natural selection. Nature 406, 985-988

37 Yoshida, T. et al. (2003) Rapid evolution drives ecological dynamics in a predator-prey system. Nature 424, 303-306

38 Hassell, M.P. (2000) The Spatial and Temporal Dynamics of HostParasitoid Interactions, Oxford University Press

39 Falconer, D.S. and Mackay, T.F.C. (1996) Introduction to Quantitative Genetics, Longman

40 Rousset, F. (1997) Genetic differentiation and estimation of gene flow from $F$-statistics under isolation by distance. Genetics $145,1219-1228$

41 Beaumont, M.A. (2003) Estimation of population growth or decline in genetically monitored populations. Genetics 164, 1139-1160

42 Zhang, X.S. et al. (2004) Redistribution of gene frequency and changes of genetic variation following a bottleneck in population size. Genetics 167, 1475-1492

43 Nunney, L. and Cambell, K.A. (1993) Assessing minimum viable population size: demography meets population genetics. Trends Ecol. Evol. 8, 234-239

44 Lande, R. (1995) Mutation and conservation. Conserv. Biol. 9, 782-791

45 Lynch, M. et al. (1995) Mutation accumulation and the extinction of small populations. Am. Nat. 146, 489-518

46 Fernández, J. and Toro, M.A. (2006) A new method to estimate relatedness from molecular markers. Mol. Ecol. doi:10.1111/j.1365294X.2006.02873.x

47 Ritland, K. (2000) Marker-inferred relatedness as a tool for detecting heritability in nature. Mol. Ecol. 9, 1195-1204

48 Umina, P.A. et al. (2005) A rapid shift in a classic clinal pattern in Drosophila reflecting climate change. Science 308, 691-693 
49 Cook, L.M. (2003) The rise and fall of the carbonaria form of the peppered moth. Q. Rev. Biol. 78, 399-417

50 Raymond, M. et al. (2001) Insecticide resistance in the mosquito Culex pipiens: what have we learned about adaptation? Genetica 112-113, 287-296

51 Barton, N.H. and Hewitt, G.M. (1989) Adaptation, speciation and hybrid zones. Nature 341, 497-503

52 Moorcroft, P.R. et al. (1996) Density-dependent selection in a fluctuating ungulate population. Proc. R. Soc. B 263, 31-38

53 Morin, P.A. et al. (2005) SNPs in ecology, evolution and conservation. Trends Ecol. Evol. 19, 208-216

54 Slate, J. et al. (2000) Inbreeding depression influences lifetime breeding success in a wild population of red deer (Cervus elaphus). Proc. R. Soc. B 267, 1657-1662

55 Acevedo-Whitehouse, K. et al. (2003) Inbreeding: disease susceptibility in California sea lions. Nature 422, 35

56 Hansson, B. and Westerberg, L. (2002) On the correlation between heterozygosity and fitness in natural populations. Mol. Ecol. 11, 2467-2474

57 Balloux, F. et al. (2004) Does heterozygosity estimate inbreeding in real populations? Mol. Ecol. 13, 3021-3031

58 Watt, W.B. (1977) Adaptation at specific loci. I. Natural selection on phosphoglucose isomerase of Colias butterflies: biochemical and population aspects. Genetics 87, 177-194

59 Wilding, C.S. et al. (2001) Differential gene exchange between parapatric morphs of Littorina saxatilis detected using AFLP markers. J. Evol. Biol. 14, 611-619

60 Loeschcke, V. et al. (2004) Ecologically relevant stress resistance: from microarrays and quantitative trait loci to candidate genes - A research plan and preliminary results using Drosophila as a model organism and climatic and genetic stress as model stresses. J. Biosci. $29,503-511$

61 Slate, J. (2005) Quantitative trait locus mapping in natural populations: progress, caveats and future directions. Mol. Ecol. 14, 363-379

62 Fitzpatrick, M.J. et al. (2005) Candidate genes for behavioural ecology. Trends Ecol. Evol. 20, 96-104

63 Oleksiak, M.J. et al. (2002) Variation in gene expression within and among natural populations. Nat. Genet. 32, 261-266
64 Thomas, M.A. and Klapper, R. (2004) Genomics for the ecological toolbox. Trends Ecol. Evol. 19, 439-445

65 Hanski, I. and Saccheri, I.J. (2006) Molecular-level variation affects population growth in a butterfly metapopulation. PLoS Biol. 4, e129

66 Wallace, B. (1975) Hard and soft selection revisited. Evolution 29, $465-473$

67 Wallace, B. (1981) Basic Population Genetics, Columbia University Press

68 Kelley, J.L. et al. (2005) Soft and hard selection on plant defence traits in Arabidopsis thaliana. Evol. Ecol. Res. 7, 287-302

69 Ridley, M. (2003) Evolution, Blackwell Science

70 Hanski, I. (1998) Metapopulation dynamics. Nature 396, 41-49

71 Olivieri, I. and Gouyon, P.H. (1997) Evolution of migration rate and other traits: the metapopulation effect. In Metapopulation Biology: Ecology, Genetics, and Evolution (Hanski, I.A. and Gilpin, M.E., eds), pp. 293-324, Academic Press

72 Hanski, I. et al. (2004) Variation in migration rate among individuals maintained by landscape structure. Ecol. Lett. 7, 958-966

73 Haag, C.R. et al. (2005) A candidate locus for variation in dispersal rate in a butterfly metapopulation. Proc. $R$. Soc. B 272, 2449-2456

74 Hanski, I. and Singer, M.C. (2001) Extinction-colonization dynamics and host-plant choice in butterfly metapopulations. Am. Nat. 158, 341-353

75 Hanski, I. and Heino, M. (2003) Metapopulation-level adaptation of insect host plant preference and extinction-colonization dynamics in heterogeneous landscapes. Theor. Popul. Biol. 64, 281-290

76 Levins, R. (1975) Evolution in communities near equilibrium. In Ecology and Evolution of Communities (Cody, M.L. and Diamond, J.M., eds), pp. 16-50, Harvard University Press

77 León, J.A. and Charlesworth, B. (1978) Ecological versions of Fisher's fundamental theorem of natural selection. Ecology 59, 457-464

78 May, R.M. and Anderson, R.M. (1983) Epidemiology and genetics in the coevolution of parasites and hosts. Proc. R. Soc. B 219, 281-313

79 Fenner, F. (1983) Biological control, as exemplified by smallpox eradication and myxomatosis. Proc. R. Soc. B 218, 259-285

80 Trout, R.C. et al. (1992) The effect on a British wild rabbit population (Oryctolagus cuniculus) of manipulating myxomatosis. J. App. Ecol. 29, 679-686

\section{ScienceDirect collection reaches six million full-text articles}

Elsevier recently announced that six million articles are now available on its premier electronic platform, ScienceDirect. This milestone in electronic scientific, technical and medical publishing means that researchers around the globe will be able to access an unsurpassed volume of information from the convenience of their desktop.

ScienceDirect's extensive and unique full-text collection covers over 1900 journals, including titles such as The Lancet, Cell, Tetrahedron and the full suite of Trends and Current Opinion journals. With ScienceDirect, the research process is enhanced with unsurpassed searching and linking functionality, all on a single, intuitive interface.

The rapid growth of the ScienceDirect collection is due to the integration of several prestigious publications as well as ongoing addition to the Backfiles - heritage collections in a number of disciplines. The latest step in this ambitious project to digitize all of Elsevier's journals back to volume one, issue one, is the addition of the highly cited Cell Press journal collection on ScienceDirect. Also available online for the first time are six Cell titles' long-awaited Backfiles, containing more than 12,000 articles highlighting important historic developments in the field of life sciences.

The six-millionth article loaded onto ScienceDirect entitled "Gene Switching and the Stability of Odorant Receptor Gene Choice" was authored by Benjamin M. Shykind and colleagues from the Dept. of Biochemistry and Molecular Biophysics and Howard Hughes Medical Institute, College of Physicians and Surgeons at Columbia University. The article appears in the 11 June issue of Elsevier's leading journal Cell, Volume 117, Issue 6, pages 801-815. 